\title{
A dose of vitamins and a pinch of salt
}

The study, published last week, purporting to show that children's IQ can be increased by dietary supplements, is an unhappy piece of work. The association could well be due to chance.

DOEs intellectual power grow from the neck of a bottle of pills? With all the ballyhoo about the effect of dietary supplements on IQ - discussed also on pages 2 and 5 of this issue, and in the main mercifully so far confined to Britain - one might be forgiven for supposing that it does. The scientific controversy centres on a "symposium" issue of the journal Personality and Individual Differences (12, 329-366; 1991), which contains studies by S. J. Schoenthaler, H. J. Eysenck, J. Yudkin and colleagues purporting to show that a modest dietary supplement of 23 vitamins and minerals taken on a sustained basis leads to a significant elevation of non-verbal IQ.

The specific importance of non-verbal tests in this context is that the abilities they tap are thought to be less affected by schooling and to be a better reflection of a person's underlying potential than those represented in verbal tests. The claimed improvement is 3.7 points using the well-known WISC-R test over a 13-week experimental period. The effect is reported only for a supplement of 100 per cent of recommended daily allowance (RDA), however, not for groups given 50 per cent or 200 per cent of RDA, and no claim is made for improvements in verbal IQ or full-scale IQ. The RDAs in question, of 12 vitamins and six minerals, are set at the US levels, rather than the lower UK levels. For four further minerals included in the treatmen, and for vitamin K, no RDAs have been established.

Of principal interest is the large-scale study by Schoenthaler et al. which involved three treatment groups and a placebo group in a double-blind design. Twenty-nine scores on cognitive tests of one kind or another were analysed, but three of these (possibly five - the text is not clear) are summary scores, and not independent of the others. Three contrasts (each treatment group with placebo) were available for each test, resulting in a total of 87 contrasts. Seven of these yielded "significant" results - the object assembly and coding subtests of WISC-R and the non-verbal IQ score to which they contribute, the arithmetic subtest (which contributes to verbal IQ), and the reading and comprehension tests from the Comprehensive Test of Basic Skills, plus the total score from this battery.

Other non-verbal tests, including three further subscales of WISC-R, Raven's Matrices and the Matrix Analogies Test, produced no significant effect, nor did five other verbal subtests of WISC-R. That is to say, four of the seven effects reported to be signi- ficant are related to verbal IQ or achievement tests, three to non-verbal IQ; looking only at scores that are independent of each other, five out of 78 contrasts are significant, of which three relate to verbal IQ, two to non-verbal. By chance, about one result in 20 is expected to reach the criterion for significance: given the tendency of scores on these tests to intercorrelate highly, the results are too close to what might be expected by chance to inspire confidence in the findings.

The results, however, are not presented in quite this way. Some of the time significance tests are not presented at all, but are replaced by rank orderings of average scores in the various groups, with a passing reference to significance tests in the text. There is no overall multivariate analysis of variance done, which, because the various test scores may be expected to be heavily intercorrelated, severely limits the interpretability of results. Instead, many univariate statistical tests are applied, leaving the sense that the data have been quarried for support for the desired effect rather than analysed on a statistical model. There is the reek of conviction driving analysis and presentation, of Schoenthaler et al. striving to make a case, rather than coolly analysing experimental results. Where results do not fit the hypothesis, special pleading comes to the rescue. For instance:

The absence of a significant relationship using the MAT [Matrix Analogies Test - a non-verbal test] was not totally unexpected. Since the test is given in a group setting, the opportunity for students to take the test lightly and rush through it was present. The large standard deviation suggests a number of students did just that. However, the trend between the 4 groups was the same as found on the nonverbal half of the WISC-R. More specifically, the placebo group did the worst and the $100 \%$ RDA group the best, making the MAT supportive of the main findings.

A more blatant piece of post-hoccery is scarcely to be found. It is one thing to analyse data every which way to search for hypotheses for testing on a replication sample, quite another to present several forms of analysis chosen to suit a preferred hypothesis as proof of that hypothesis.

There are plenty of other holes in the information presented. For instance the precise basis on which raw scores were converted to IQs - important because WISC-R uses age-based norms, and in their teenage years children are getting better at the skills tested - is not given; nor is information on actual test score statistics for the four experimental groups; nor are details of correlations amongst the various measures.
The results of assays of blood samples, taken as part of the study, were not available at the time this research was published (other work in the symposium issue suggests that any effect of dietary supplementation is reflected in blood nutrient concentrations). And the results of a parallel study undertaken in England (rather than California) were not ready. So why publish now? No front-rank journal would contemplate publication in this form of incomplete findings bearing on such an important issue.

This is not to say that there is no phenomenon worthy of attention. Other, smallerscale work described in the same symposium suggests that vitamin and mineral deficiency may be an important determinant of IQ and behaviour amongst delinquent adolescents, and that remedying the deficiency has desirable outcomes. It is not difficult to believe that some children would benefit in their intellectual development from dietary supplements or better eating habits, and there are enough hints in the data consistent with this view to make it worth seeking means of identifying who they may be. But the recommendation made by Eysenck, that the best policy would be to administer a 100 per cent RDA dose to all children on the basis of the present evidence is at best premature. What, for instance, of those children who did worse than expected in the treatment groups?

Schoenthaler et al. interpret their results as showing that dietary supplementation improved non-verbal intelligence estimates by a minimum of 6 points, an average of 11 points and a maximum of 21 points. This is so only for subjects preselected for showing large gains in scores over the experimental period. This style of interpretation of difference scores is psychometrically naive and thoroughly misleading. The authors write: "no change is expected in most students with dramatic changes appearing in others", but no evidence is presented of such a marked bimodality in the data.

The purported significant elevation of IQ as a result of vitamin treatment is a feeble thing at best - it is neither robust across different tests, nor large, and the claim that it relates to non-verbal rather than verbal tests is not supported by the data. Parents who buy dietary supplements because they have been told they will make their children more intelligent are, on this evidence, being misled.

Steve Blinkhorn

Steve Blinkhorn is managing director of Psychometric Research and Development Ltd, Brewmaster House, The Maltings, St Albans, Hertfordshire AL1 3HT, UK. 